\title{
Transcriptome analysis of potential candidate genes and molecular pathways in colitis-associated colorectal cancer of Mkp-1-deficient mice
}

Ahmed Hammad ${ }^{1 \dagger}$, Zhao-Hong Zheng ${ }^{2 \dagger}$, Akhileshwar Namani ${ }^{1,3}$, Mohamed Elshaer ${ }^{1}$, Xiu Jun Wang ${ }^{2}$ and Xiuwen Tang ${ }^{1 *}$

\begin{abstract}
Background: The nuclear phosphatase mitogen-activate protein kinase phosphatase-1 (MKP-1) is a key negative regulator of the innate immune response through the regulation of the biosynthesis of proinflammatory cytokines. In colorectal cancer (CRC), which is induced mainly by chronic inflammation, Mkp-1 overexpression was found in addition to disturbances in Mkp-1 functions, which may play a role in cancer development in different types of tumors. However, the potential molecular mechanisms by which Mkp-1 influences CRC development is not clear. Here, we performed global gene expression profiling of Mkp-1 KO mice using RNA sequencing (RNA-seq) to explore the role of Mkp-1 in CRC progression using transcriptome analysis.

Methods: Azoxymethane/dextran sodium sulfate (AOM/DSS) mouse models were used to examine the most dramatic molecular and signaling changes that occur during different phases of CRC development in wild-type mice and Mkp-1 KO mice. Comprehensive bioinformatics analyses were used to elucidate the molecular processes regulated by Mkp-1. Differentially expressed genes (DEGs) were identified and functionally analyzed by Gene Ontology (GO), Kyoto Enrichment of Genes and Genomes (KEGG). Then, protein-protein interaction (PPI) network analysis was conducted using the STRING database and Cytoscape software.

Results: Persistent DEGs were different in adenoma and carcinoma stage (238 \& 251, respectively) and in WT and MKp-1 KO mice (221\& 196, respectively). Mkp-1 KO modulated key molecular processes typically activated in cancer, in particular, cell adhesion, ion transport, extracellular matrix organization, response to drug, response to hypoxia, and response to toxic substance. It was obvious that these pathways are closely associated with cancer development and metastasis. From the PPI network analyses, nine hub genes associated with CRC were identified.

Conclusion: These findings suggest that MKp-1 and its hub genes may play a critical role in cancer development, prognosis, and determining treatment outcomes. We provide clues to build a potential link between Mkp-1 and colitis-associated tumorigenesis and identify areas requiring further investigation.
\end{abstract}

Keywords: Mkp-1, Colitis, Colorectal cancer, RNA sequencing, Biomarker

\footnotetext{
* Correspondence: xiuwentang@zju.edu.cn

${ }^{+}$Ahmed Hammad and Zhao-Hong Zheng contributed equally to this work. 'Department of Biochemistry and Department of Thoracic Surgery of The First Affiliated Hospital, Zhejiang University School of Medicine, Hangzhou 310003, People's Republic of China

Full list of author information is available at the end of the article
} 


\section{Introduction}

Colorectal cancer (CRC) is the third most common malignancies worldwide [1], with more than 1.2 million new cases diagnosed annually [2]. According to Cancer statistics in China, 2015, there were approximately 376, 000 new patients with CRC and 191,000 new CRC death, accounting for a fifth of malignant tumor incidence and mortality [3].

CRC metastasis is a common phenomenon and the major lethal cause of CRC, as approximately $50 \%$ of patients with CRC die within 5 years due to extensive metastasis [4]. CRC developed through a multistep process from normal epithelium to adenoma and adenocarcinoma, which can eventually metastasize to different organs [5]; it was shown that the transition of CRC cells from early stages to late stages (metastasis) is associated with a significant change in the cellular gene expression profile [6]. These genetic alterations have led to the emergence of the novel strategies for personalized medicine programs $[7,8]$. Moreover, the correlation between colorectal inflammation and increased colitis-associated tumorigenesis has been identified in some epidemiological and clinical studies $[9,10]$, which is characterized by high mortality and is closely associated with inflammatory bowel disease [11].

Mitogen-activated protein kinase phosphatases (MAPK phosphatases, MKPs) are a family of proteins that have been identified as major negative regulators of MAPK activities, which act as potential regulators of cell growth and survival in physiological and pathological processes [12]. Specifically, Mkp-1, also named dual-specific phosphatase 1 , is a nuclear phosphatase and a key negative regulator in the innate immune response [13, 14], by deactivating p38 and c-Jun N-terminal kinases by dephosphorylating threonine and tyrosine, which play pivotal regulatory roles in the biosynthesis of proinflammatory cytokines. Notably, in colon cancer, Mkp-1 was overexpressed in the early phases of carcinogenesis [15, 16], and it impaired the response to cetuximab-based treatment in patients with metastatic colon cancer [17]. Furthermore, the therapeutic effect of camptothecin in colon cancer cells was improved through inhibition of Mkp-1 activity [18]. These studies suggest that Mkp-1 may be an attractive target for colon cancer therapy [15]. The involvement of Mkp-1 in cancer therapy resistance has also gained prominence, making $\mathrm{Mkp}-1$ a potential target for anti-cancer therapy [12].

Indeed, the role of Mkps in cancer has been less welldefined despite increasing evidence that disturbances in Mkp-1 function may play a role in cancer development. According to recently published papers, there are controversial roles of $\mathrm{Mkp}-1$ in cancer development and progression. Although some studies have shown that the gain of Mkps expression has been associated with drug resistance, cancer progression, and poor patient prognosis [19], others have shown that the loss of Mkps induces cancer development or progression due to the loss of negative regulation of MAPKs [12].

Conclusively, the main cause of CRC is the disturbance in immune responses and some metabolic pathways, in addition to the main roles of $\mathrm{Mkp}-1$ involved in the regulation of immune responses. We aim to highlight the role of $\mathrm{Mkp}-1$ in CRC in this study using transcriptome analyses associated with bioinformatics data mining tools, using RNA-seq, which provide an opportunity to simultaneously analyze a huge number of genes/targets, and explore the potential genes and molecular signaling pathways regulated by $\mathrm{Mkp}-1$.

\section{Material and methods Chemicals}

Unless otherwise stated, all chemicals purchased from Sigma-Aldrich Co., Ltd. (St. Louis, MO, USA). Antibodies were obtained from Santa Cruz Biotechnology (Dallas, TX, USA). DSS (36-50 kDa) was obtained from MP Biomedicals (Aurora, OH, USA).

\section{Animals and animal ethics}

BALB/c background WT mice were purchased from Shanghai Laboratory Animal Center (Chinese Academy of Sciences, Shanghai, China). After obtaining the permission from Bristol-Myers Squibb Co. (New York, NY, USA), Professor Andrew R. Clark (University of Birmingham, UK) provided us $M k p-1^{-/-}$mice on a C57BL/ 6 background [20]. BALB/c background $M k p-1^{-1-}$ mice were produced by eight back-crossings of $\mathrm{C} 57 \mathrm{BL} / 6$ $M k p-1^{-/-}$mice with BALB/c WT mice. Genotypes of $M k p-1^{-/-}$mice were routinely identified by RT-PCR and western immunoblotting. Mice were maintained in the cages (VM370B64S9 cages with size 370x157x180 mm, Suhangtech, Jiangsu, China) in groups of four under standard conditions of ventilation, temperatures $(22 \pm 30$ $\mathrm{C}$ ), and lighting (light/dark: $13 \mathrm{~h} / 11 \mathrm{~h}$ ) and kept under observation for 1 week prior to experimentation. Drinking water and standard pellets diet were provided ad libitum throughout the study. All animal experiments were performed with the approval of the Laboratory Animal Ethics Committee of Zhejiang University. Animal studies were conducted in compliance with the ARRIVE guidelines [21, 22]. All the studies were performed in accordance with relevant guidelines and regulations. A complete ARRIVE Guidelines checklist is included in Additional file 1.

\section{Induction of colitis-associated CRC}

The azoxymethane (AOM)-initiated and dextran sodium sulfate (DSS)-promoted mice model has been widely used to simulate the pathogenesis observed in patients 
with CRC [23]. In fact, carcinogenesis was induced by the injection of an AOM (inducing aberrant crypt foci caused by DNA damage) and DSS mixed in the drinking water (inducing colitis caused by inflammatory damage in the epithelial lining of the colon) in mice [24]. Therefore, two colitis-associated CRC models of adenoma and adenocarcinoma, which represent the initial and later stages of colon cancer, respectively, were induced using a AOM/DSS treatment strategy modified from that of Suzuki et al. [25] as we were previously described [26]. The protocol for the induction of adenoma stage (AS) is illustrated in Fig. 1. Briefly, 5-6-week-old male BALB/c mice were injected with $\mathrm{AOM}(10 \mathrm{mg} / \mathrm{kg}$ i.p.) once per week for the first 3 weeks. During this period, mice were exposed to drinking water containing 1.5\% DSS and were left to drink normal water for 3 weeks. For the carcinoma stage (CS), 5-6-week-old-old male mice were also injected with AOM $(10 \mathrm{mg} / \mathrm{kg}$ i.p.), exposed to drinking water containing $2 \%$ DSS for 1 week, and then left to drink normal water for 2 weeks. This treatment was repeated for three additional cycles. Finally, the mice were left to drink water containing $2 \%$ DSS for 1 week, followed by normal water for 9 weeks. At the end of each period of AS and CS, mice were sacrificed by cervical dislocation. The colon was dissected as described previously [13], and hematoxylin and eosin (H\&E) staining was performed for histopathological analysis of tissue sections $(3-\mu \mathrm{m})[27,28]$.

\section{RNA-seq analysis}

RNA-seq analysis and library were described previously [26]. Indeed, The present study involved two main categories including wild type that subdivided to four subgroups; wild type control (WT-C) for adenoma stage, wild type adenoma stage (WT-AS), wild type control (WT-C) for carcinoma stage, wild type carcinoma stage (WT-CS), which we used them in our previous work but in different purposes [26], and Mkp-1 KO type that subdivided to four subgroups; Mkp-1 KO control (Mkp$\left.1^{-/} \mathrm{C}\right)$ for adenoma stage, Mkp-1 KO adenoma stage $\left(M k p-1^{-/-} \mathrm{AS}\right)$, Mkp-1 KO control $\left(M k p-1^{-/-} \mathrm{C}\right)$ for carcinoma stage, Mkp-1 KO carcinoma stage $\left(M k p-1^{-/-} \mathrm{C}\right.$ $S$ ). At the end of the experiment, Animals ( $n=3$ for each group) were sacrificed and distal colonic epithelial tissue samples from each group were dissected and subjected to total RNA extraction using TRIzol reagent (Invitrogen, Carlsbad, CA) according to the manufacture's instruction. RNA quality was assessed using an Agilent Bioanalyzer (Agilent Technologies, Palo Alto, CA). The RNA was sheared and reverse transcribed using random primers to obtain cDNA, which was used for library construction. Illuminam RNA-Seq libraries were subsequently performed using the SMARTer Stranded RNASeq Kit (Clontech, Mountain View, CA) according to the manufacturer's instructions. All statistical analyses were carried out with HTSeq v0.6.1; after removing transcriptionally inactive genes (read count per million $<1$ ) from

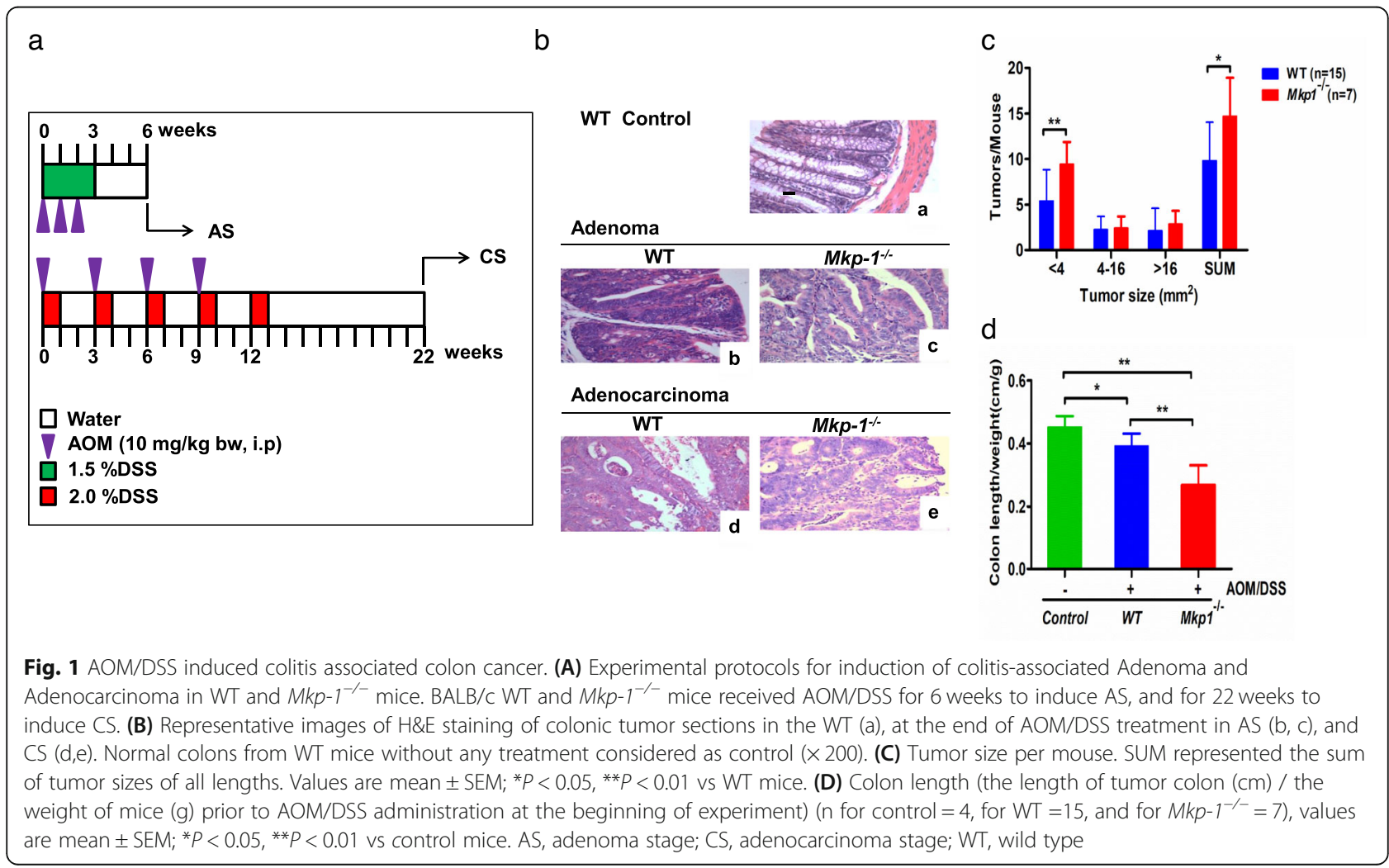


raw RNA sequencing gene counts, we obtained high confidence gene counts. For gene expression analysis, the matched reads were calculated and then normalized to reads per kilobase of exon model per million mapped reads (FPKM) [29, 30]. The RNA-seq data of raw and processed files have been deposited in NCBI Gene Expression Omnibus, accession number GSE164960.

\section{Screening for differentially expressed genes (DEG)}

The DEGs of WT-AS vs. WT-C, WT-CS vs. WT-C, $M k p-1^{-/-}$AS vs. $M k p-1^{-/-}$C, and $M k p-1^{-/-}$CS vs. $M k p-$ $1^{-/-} \mathrm{C}$ were identified using the $\mathrm{R}$ statistical software package (www.r-project.org). The cutoff criteria were $|\log F C| \geq 1.5$ and adjusted $P$-value $<0.05$. Moreover, overlapping DEGs between different groups have been identified by UpSet plots [31].

\section{Functional enrichment analysis}

The enrichment analyses using GO terms (http://www. geneontology.org/) and KEGG pathways (http://www. genome.jp/kegg/) data for gene sets were performed using the NIH Database for Annotation, Visualization and Integrated Discovery (DAVID) web tool [32]. This tool can provide a functional interpretation of huge gene lists derived from genomic studies. A Benjamini $p$-value of $<0.05$ was used in the analysis.

\section{Protein-protein interaction (PPI) network analysis}

To further investigate the molecular mechanism of knockout in CRC, specific DEGs of $M k p-1^{-/-}$groups were used to construct the PPI network by utilizing the biological online database tool (Search Tool for the Retrieval of Interacting Genes, STRING, http:// stringdb.org) [33] to identify and explore the interactions among them. A combined score of $>0.4$ (high confidence score) was considered significant, and the PPI network was visualized using Cytoscape software (Version3.5.1) [34]. The hub genes/proteins, a small number of crucial nodes for the protein interactions in the PPI network, were chosen with a centrality degree $>5$.

\section{Cluster analysis for hub genes}

The expression profile of hub genes was performed using heatmaper, a web-based tool [35].

\section{Survival analysis}

Hub genes in various cohorts were subjected to a survival analysis using PROGgeneV2 [36] and PrognoScan [37].

\section{Statistical analyses}

Statistical analyses were carried out using Stata7 for Windows (Stata Corp LLC, College Station, TX, USA). Student's t-test was used to compare the two groups.
Groups of more than two were compared using one-way ANOVA followed by Bartlett's test. Spearman's correlation was used to analyze the two ranked variables. One mouse was classified as one experimental unit. A $P$-value $<0.05$ was considered statistically significant.

\section{Results}

Mkp-1 deficiency promotes tumorigenesis in AOM/DSSinduced colitis-associated CRC

Treatment of $M k p$-1-deficient mice with AOM/DSS (Fig. 1A) triggered the colitis, which was confirmed by a significant decrease in the length of colon in $\mathrm{KO}$ mice compared to WT one (Fig. 1D). Histopathological examination using H\&E staining showed that AOM/DSS treatment for 6 weeks induced adenoma stage in WT and KO mice compared to normal WT colon, representing the early stage of tumorigenesis. Treatment for 22 weeks induced adenocarcinoma stage, representing the late stage of tumorigenesis (Fig. 1B). In addition, the number of smaller tumors $\left(<4 \mathrm{~mm}^{3}\right)$ per mouse in $\mathrm{KO}$ $(P$ value $<0.01)$ mice compared to WT mice increased; the numbers of medium-sized $\left(4-16 \mathrm{~mm}^{3}\right)$ and large (> $16 \mathrm{~mm}^{3}$ ) tumors per mouse were similar in the KO and WT mice (Fig. 1C).

\section{Novel molecular pathways identified in carcinoma stages} RNA-Seq provides an opportunity to simultaneously analyze a huge number of genes and distinguish between molecular pathways of early stage of CRC (adenoma) and late stage of CRC (adenocarcinoma), thus we analyzed the profiles of DEGs between diseased mice and control mice following the cutoff criteria fold-change $|\operatorname{logFC}|>1.5$ and $P$-value $<0.05$ by ANOVA test. A library with size-normalized count for the specimen was generated by making volcano plots for the DEGs (Additional file 2: sFig. 1).

Data from RNA-seq were analyzed to obtain overlapping DEGs in adenoma and carcinoma stages. We identified 94 upregulated and 144 downregulated common DEGs in adenomas (553 DEGs in wild type divided into 213 upregulated and 340 downregulated and 390 DEGs in $\mathrm{KO}$ type divided to 158 upregulated \& 232 downregulated) (Fig. 2a). In carcinomas, 106 upregulated and 145 downregulated DEGs overlapped (421 DEGs in wild type divided into 210 upregulated \& 211 downregulated, and 535 DEGs in KO type divided into 217 upregulated \& 318 downregulated) (Fig. 2b). Indeed, the numbers of persistent DEGs in carcinoma is slightly higher than in adenoma, showing that the progression of CRC affects the profile of expressed genes. (All the gene IDs and fold changes are listed in Additional files 3 and 4).

As we mentioned above, CRC metastasis is a common phenomenon and the major cause of death, and the transition of CRC cells from early stages to late stages 

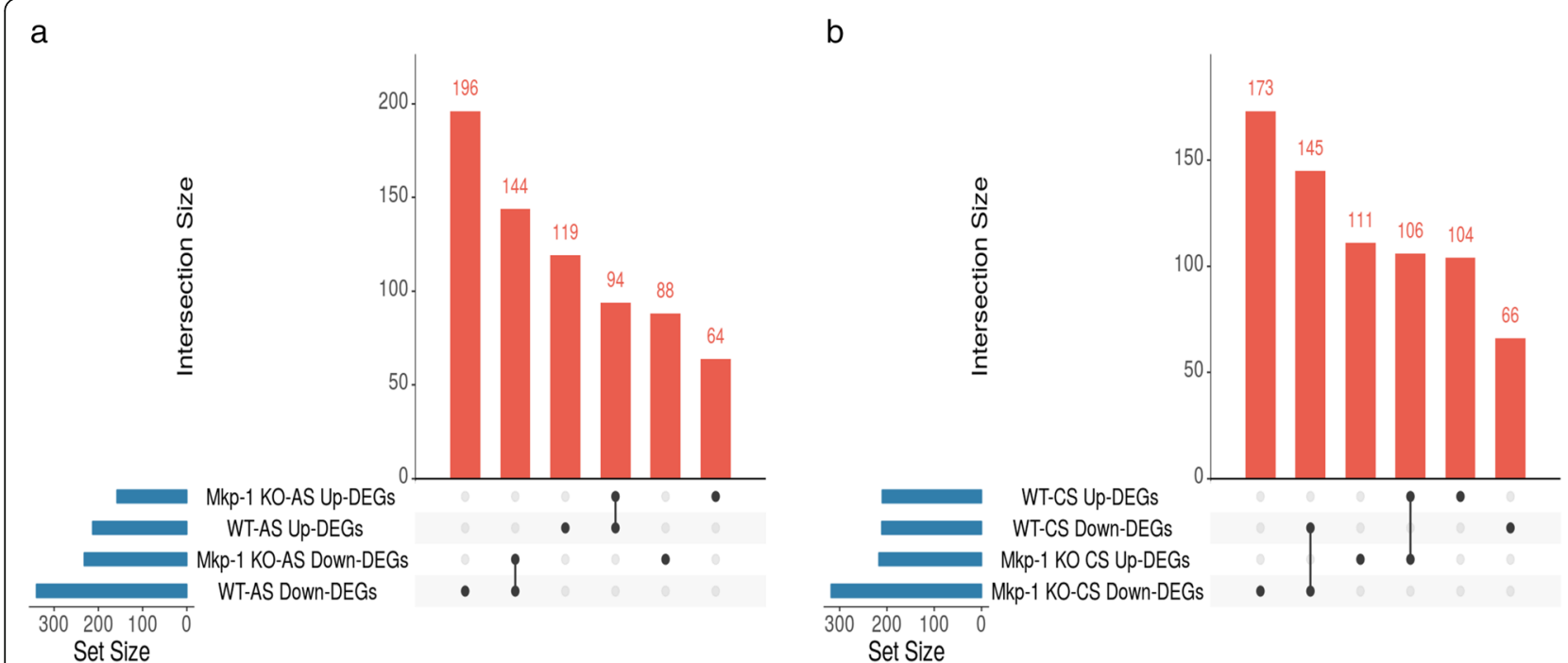

Set Size

Fig. 2 DEGs of early and late stages of tumors in the colon of mice administered with AOM/DSS. UpSet plots showing upregulated DEGs (Up-DEGs) and downregulated DEGs (Down-DEGs) overlapping in AS and CS for WT \& KO mice. a Adenoma, AS b Carcinoma, CS. Each horizontal bar on the left represents the total number of DEGs identified in each set. Each vertical bar shows the number of genes that have been detected in each of the intersection sets, indicated by the connected points in the lower part of the plot

(metastasis) is associated with a significant change in the cellular gene expression profile. Therefore, we compared signaling pathways regulated in adenomas and carcinomas to identify the novel pathways that are considered specific for carcinoma; and the findings can be used in improving the early diagnosis and treatment of CRC.

GO analysis performed using DAVID showed that in the early stage of CRC (adenoma), the upregulated DEGs were mainly enriched in organ morphogenesis,

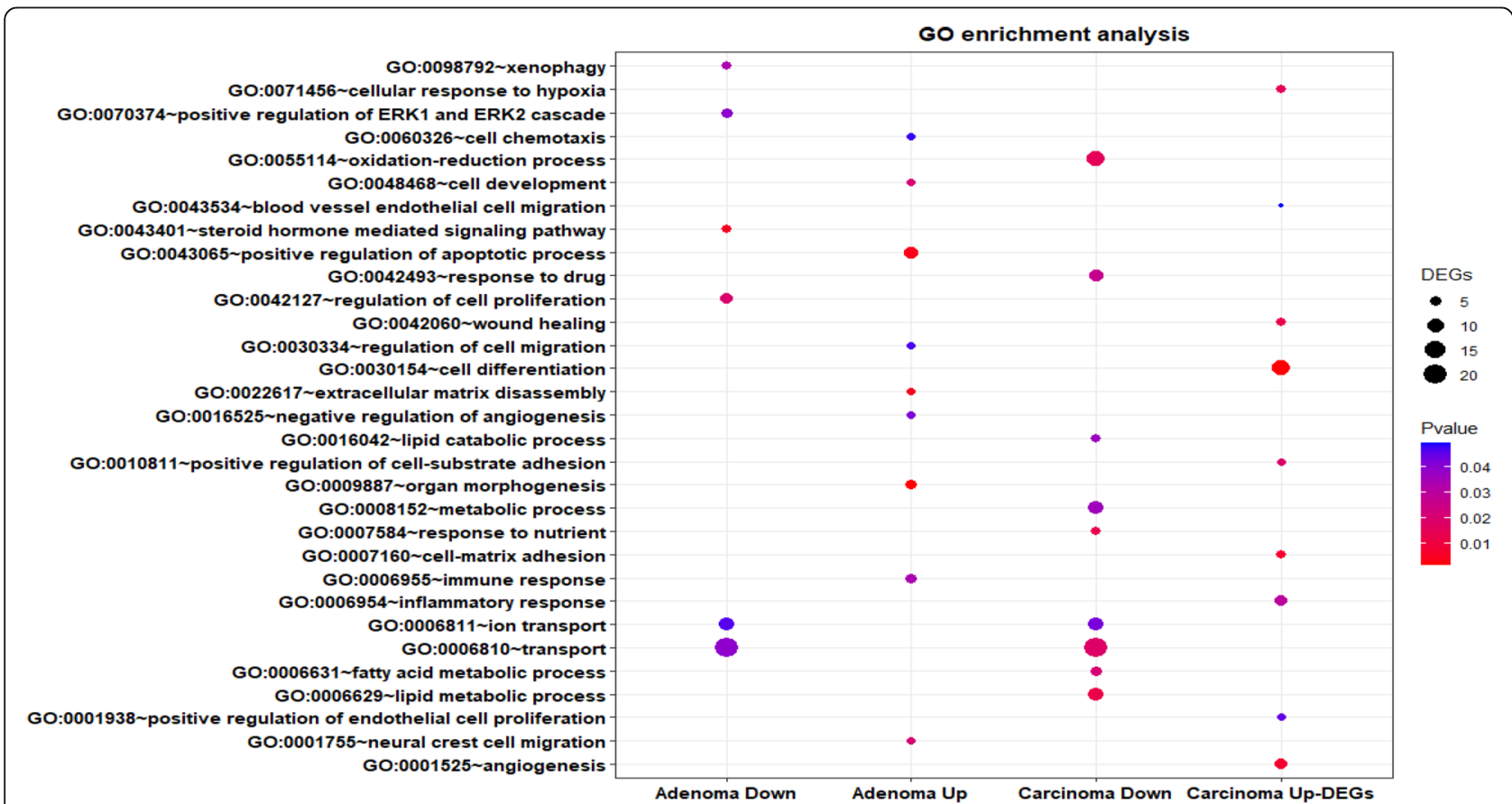

Fig. 3 GO enrichment analysis of DEGs in the different stages of CRC. Dot plots showing GO terms in early-stage of CRC adenoma and late-stage carcinoma for upregulated and downregulated DEGs. GO, Gene Ontology; the size of circle shows the number of genes while the color shows $p$-value 
positive regulation of apoptotic process, regulation of cell migration, and immune response, whereas downregulated DEGs referred to xenophagy, transport, and regulation of cell proliferation. In the carcinoma stage, the upregulated DEGs were significantly enriched in cell-matrix adhesion, cell differentiation, positive regulation of cell-substrate adhesion, and inflammatory response, while downregulated DEGs referred to transport, lipid metabolic process, oxidation-reduction process, and response to drug. These pathways in CS stages may vigorously induce the progression and metastasis of CRC (Fig. 3).

Figure 4 presents the significantly enriched KEGG [38-40] pathways of the upregulated and downregulated DEGs. For AS, the upregulated DEGs were significantly enriched in cytokine-cytokine receptor interaction, while the downregulated ones referred to circadian rhythm. In CS, the upregulated DEGs were significantly enriched in transforming growth factor-beta (TGF-beta) signaling pathway, while the downregulated ones referred to metabolic pathways and drug metabolism. These enriched terms and pathways may provide a new insight toward further research directions about the role of DEGs in colon cancer progression (Additional file 5).

\section{Mkp-1 modulates the key molecular processes typically} related to tumorigenesis and cancer progression

The DEGs identified by overlapping the DEGs specific for $M k p-1^{-/}$in both stages in $\mathrm{KO}$ mice included 221 genes, while there are 196 genes for wild-type overlapping in both stages as we previously described [26] (Fig. 5a and b).

The GO terms and KEGG pathways analyses showed that there is a significant difference between wild-type and $\mathrm{KO}$ mice, in particular, the molecular signaling in $\mathrm{KO}$ mice was highly significant in cell adhesion, extracellular matrix organization, and regulation of cell proliferation. These results suggest that the $M k p-1$ gene could modulate the regulation of key molecular processes typically related to tumorigenesis and cancer progression (Fig. 5c and d, Additional file 6) [38-40].

\section{Nine hub genes regulated by Mkp-1}

To further investigate the molecular mechanism of Mkp-1-promoted tumor growth and interactive relationships among all DEGs, we mapped the 196 DEGs to the STRING database, and validated interactions with a combined score of $>0.4$ (high confidence) were selected to construct a PPI network. The PPI network consisted of 194 nodes and 132 interactions (Fig. 6a). In the PPI network, nine proteins, including tumor necrosis factor B (Tnf), periostin (Postn), integrin alpha-8 (Itga8), actin, aortic smooth muscle (Acta2), transgelin (Tagln), growth-regulated alpha protein (Cxcl1), mimecan (Ogn), beta-enolase (Eno3), and nuclear receptor subfamily 5 group A member 2 ( $\mathrm{Nr} 5 \mathrm{a} 2)$ were strongly connected to other proteins (degree centrality>5), indicating that they

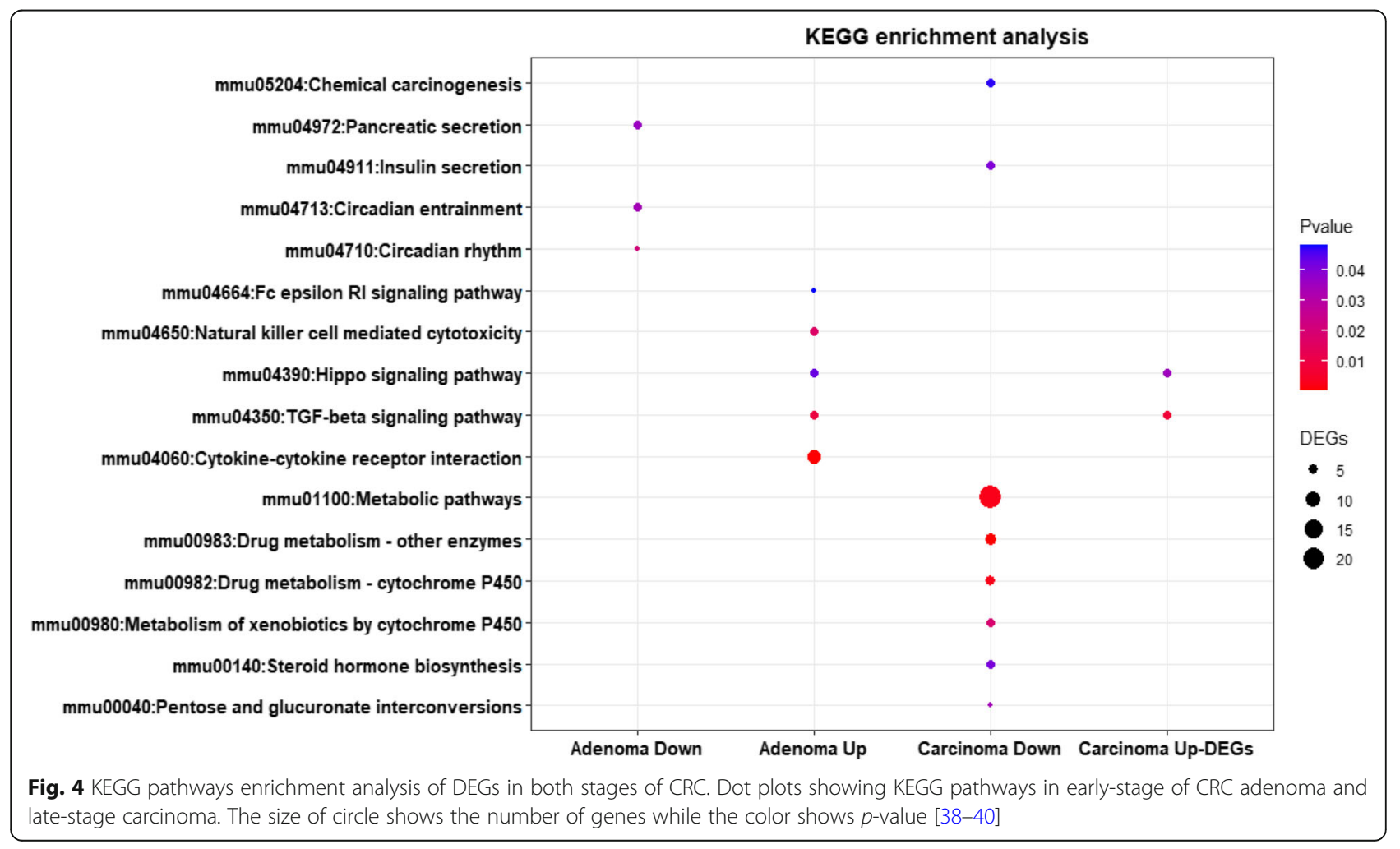




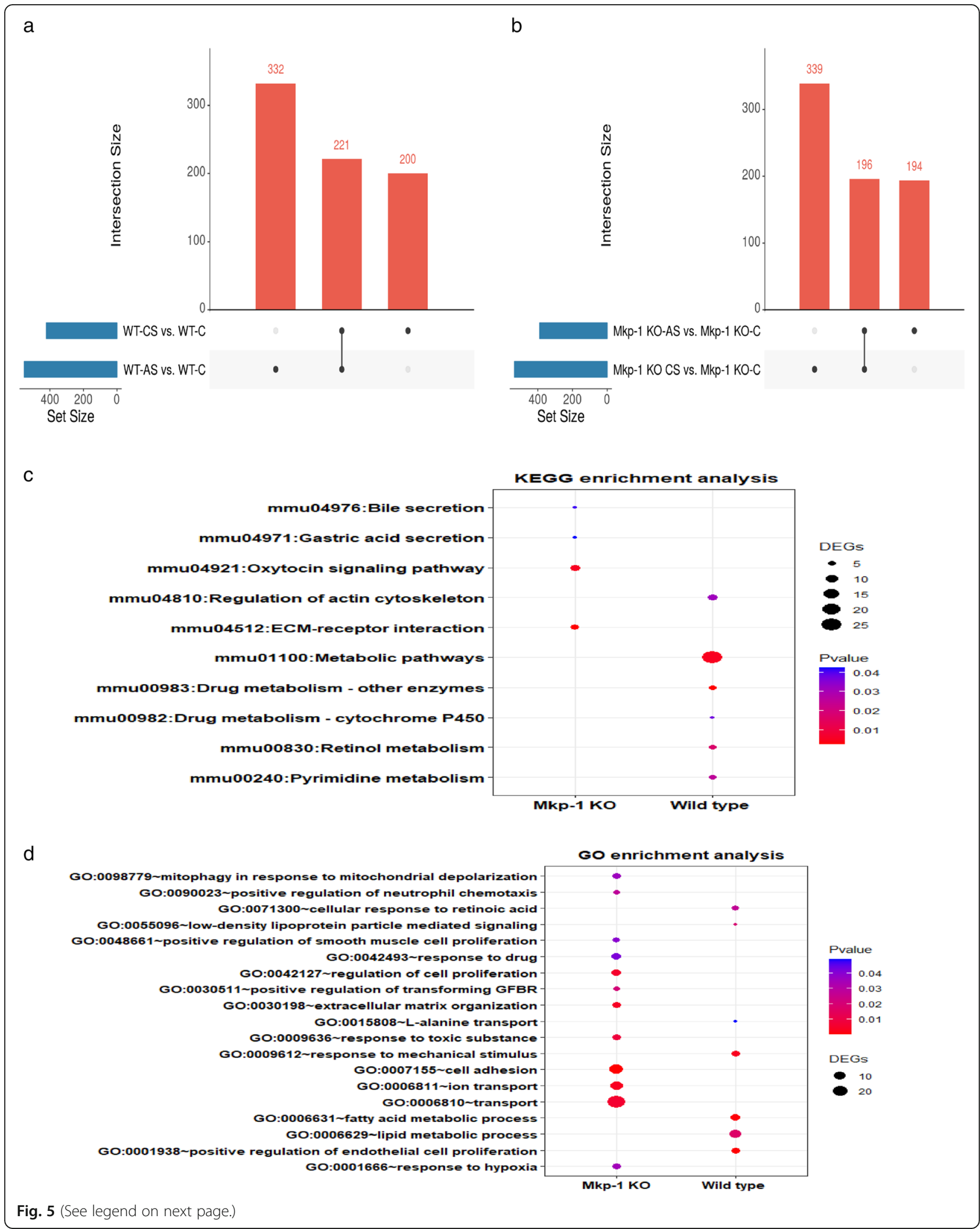


(See figure on previous page.)

Fig. 5 Mkp-1 knockdown alters gene profiling and targets in CRC. a UpSet plot showing overlapped DEGs in WT mice. b UpSet plot showing overlapped DEGs in KO mice. Each horizontal bar on the left represents the total number of DEGs identified in each set. Each vertical bar shows the number of genes that have been detected in each of the intersection sets, indicated by the connected points in the lower part of the plot. c Dot charts showing KEGG pathways comparison between KO and WT mice. $\mathbf{d}$ Dot plots showing GO terms in KO and WT mice. The size of circle shows the number of genes while the color shows p-value [38-40]

were hub genes (Fig. 6b). These hub genes might play crucial roles in the progression of CRC. The hub genes and their corresponding degrees are shown in Table 1. The GO functions and KEGG [38-40] pathways for hub genes are shown in Table 2.

\section{Nine hub genes that may be important in CRC prognosis} Using the PROGgeneV2 database, it was shown that higher mRNA expression of 9 hub genes as a signature in 2 different cohorts, GSE17536 (HR: 3.41 [1.53-7.61]) and GSE41258 (HR: 1.83[1.12-2.99]), were related to poor survival in colon cancer patients (Fig. 7). KaplanMeier analysis of the overall and disease-free survival of colorectal cancer patients was based on the PrognoScan database (http://www.prognoscan.org/) using the publicly available Gene Expression Omnibus (http://www. ncbi.nlm.nih.gov/geo) with the accession number GSE 17536, GSE17537, and GSE12945, showing that these genes might be used in CRC prognosis (Additional file 2 (sFig. 2) and Additional file 7).

\section{Discussion}

CRC can arise through the progression of adenoma, which is a consequence of genetic and epigenetic events in epithelial cells. In addition, the major causes of CRC development are Wnt/ $\beta$-catenin activation and malignant transformation of inflammatory bowel disease [41-43], leading to increased proliferation and decreased apoptosis in the intestinal tract. Moreover, several recent studies have revealed that Mkp-1, a major negative regulator of the immune response, is overexpressed in CRC and associated with poor prognosis $[15,19]$. However, the available data on the pathological role of Mkp-1 in CRC tumorigenesis are not clear and the molecular changes induced by MKp-1 in CRC have not been fully identified. Therefore, the objective of this study was to highlight the molecular signaling differences between CRC adenoma stage (AS) and carcinoma stage (CS), in addition to exploring the roles of Mkp-1 in CRC development by bioinformatics analyses using RNA-seq of WT and $\mathrm{KO}$ mice.
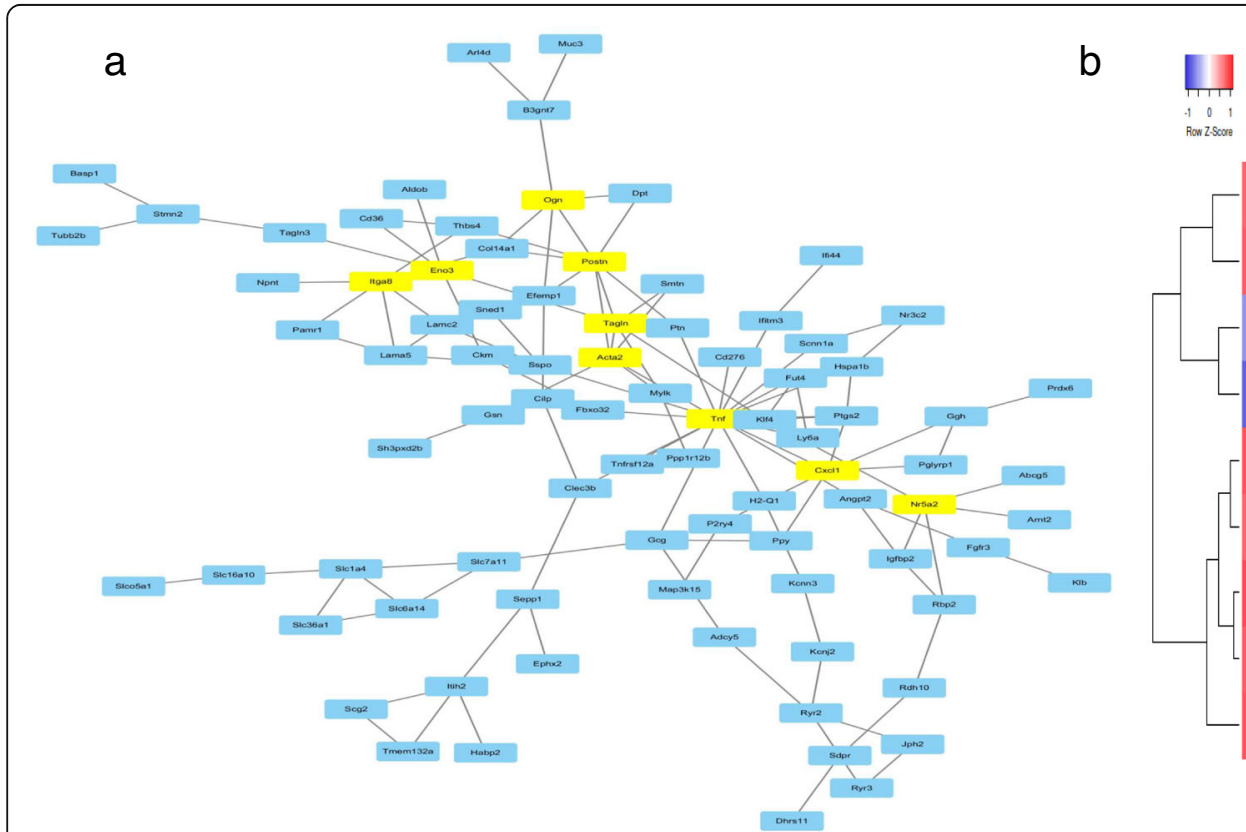

Fig. 6 a PPI network of common DEGs using STRING. The protein-protein interaction (PPI) network constructed using the differentially expressed genes common to both adenoma KO and carcinoma KO. This network contained 194 nodes and 132 interaction pairs Tnf, Postn, Itga8, Acta2, Tagln, Cxcl1, Ogn, Eno3, and Nr5a2 had high degrees. b Heatmap for hub genes showing that most of them are down-regulated in KO mice 
Table 1 The top hub genes screened by Cytoscape web tool

\begin{tabular}{llll}
\hline Name & Degree & Betweenness Centrality & Closeness Centrality \\
\hline Tnf & 17 & 0.67993243 & 0.36681223 \\
Postn & 8 & 0.17053133 & 0.28093645 \\
Itga8 & 6 & 0.04384682 & 0.21989529 \\
Acta2 & 6 & 0.19197903 & 0.32061069 \\
Tagln & 6 & 0.1451616 & 0.28668942 \\
Cxcl1 & 6 & 0.1185472 & 0.28474576 \\
Ogn & 5 & 0.07264773 & 0.23333333 \\
Eno3 & 5 & 0.13239944 & 0.23728814 \\
Nr5a2 & 5 & 0.08694301 & 0.23076923 \\
\hline
\end{tabular}

To better understand the function of DEGs in adenoma, GO analysis and KEGG pathway were performed. Upregulated genes mainly involved in biological processes consisting of organ morphogenesis, extracellular matrix disassembly, and cell development, which are essential for the initiation of tumors. Downregulated genes are also accumulated in biological processes that serve as barriers to cancer. For example, steroid hormonemediated signaling pathways such as estrogens and their receptors may in fact exert an anti-tumor effect through selective activation of pro-apoptotic signaling, inhibition of inflammatory signals, and modulation of the tumor microenvironment [44]. In addition to autophagy, xenophagy is responsible for eliminating intestinal microbiota [45] that play a key role in CRC initiation and development; therefore, xenophagy inhibits colitis and CRC initiation [46]. Moreover, disruption of circadian rhythm is involved in increasing cancer risk, chemoresistance, and progression in CRC $[47,48]$. Lastly, decreased expression of negative regulators such as PER2 and PER3 is associated with poorer differentiation, increased aggressiveness, and worse prognosis [49].

Regarding the function of DEGs in carcinoma, Upregulated genes were mainly involved in biological processes, including angiogenesis, cell migration, wound healing, cell differentiation, and inflammatory responses, which are essential for the maintenance of tumor development and metastasis [50, 51]. Downregulated genes were also found to participate in lipid metabolism, oxidation-reduction process, metabolic signaling, and drug metabolism, alteration of which is acquired by cancer to induce metabolic reprogramming and increase CRC progression [26]. Therefore, we may use the DEGs involved in the carcinoma stage found in this study to develop a novel strategy for CRC prognosis and prevent metastasis.

To address the hypothesis that Mkp-1 has critical roles in some cellular pathways required for tumor development, progression, and metastasis, we used a double strategy that combined RNA-seq and knockout technology. This strategy provided a differential expression profile of genes functionally related to Mkp-1. Here, we report that the disturbance of Mkp-1 expression in CRC induces changes in the expression levels of genes involved in tumor progression and metastasis, which may be a result of modulation of JNK, ERK and p38 activity [52]. In particular, Mkp-1 modulated key molecular processes typically activated in cancer, such as the

Table 2 GO terms and KEGG pathways for hub genes screened by string web tool

\begin{tabular}{lll}
\hline Term & Term description & FDR \\
\hline GO:0009888 & tissue development & 0.0014 \\
GO:0032722 & positive regulation of chemokine production & 0.0016 \\
GO:0009653 & anatomical structure morphogenesis & 0.0027 \\
GO:0030154 & cell differentiation & 0.0027 \\
GO:0048856 & anatomical structure development & 0.0027 \\
GO:2000343 & positive regulation of chemokine (C-X-C motif) ligand 2 production \\
GO:0050715 & positive regulation of cytokine secretion & 0.0027 \\
GO:0009887 & animal organ morphogenesis & 0.003 \\
GO:0009966 & regulation of signal transduction & 0.0035 \\
GO:2000147 & positive regulation of cell motility & 0.0048 \\
GO:0030198 & extracellular matrix organization & 0.0055 \\
KEGG pathway & & 0.0056 \\
mmu05134 & Legionellosis & 0.0225 \\
mmu04657 & IL-17 signaling pathway & 0.0228 \\
mmu04668 & TNF signaling pathway & 0.0228 \\
mmu04621 & NOD-like receptor signaling pathway & 0.0292 \\
mmu05205 & Proteoglycans in cancer & 0.0364 \\
\hline & &
\end{tabular}


a

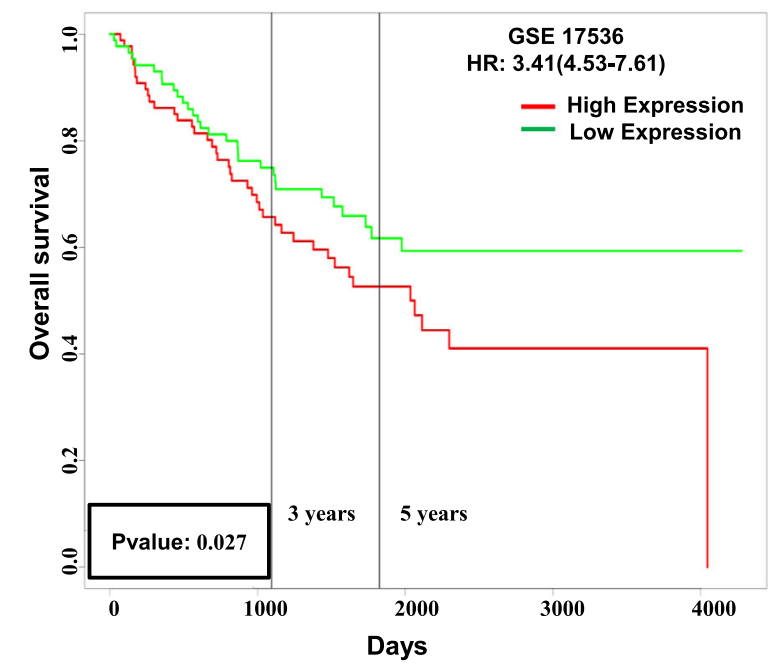

b

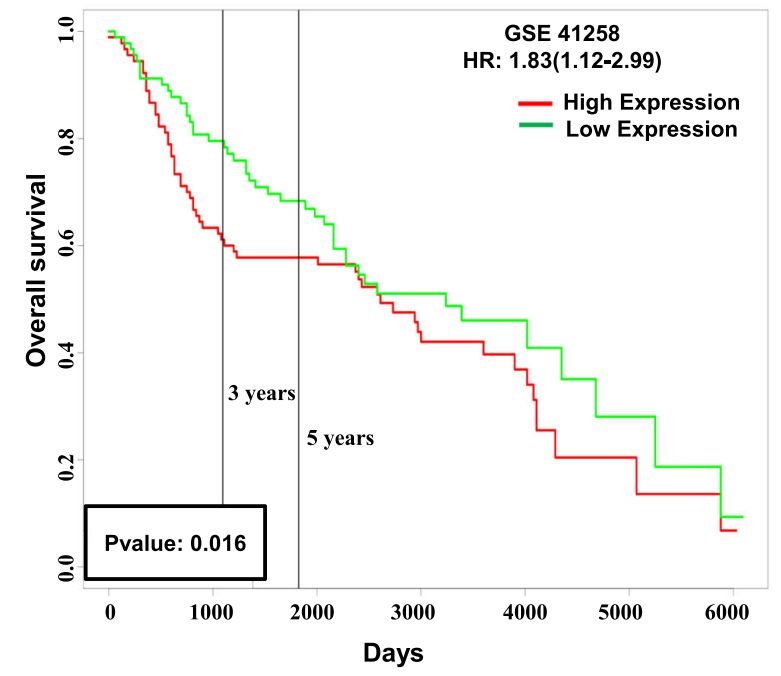

Fig. 7 Prognostic values of 9 candidate genes in different CRC cohorts. a Cohort GSE 17536. b Cohort GSE 41258

regulation of extracellular organization, proliferation, cell adhesion, transforming growth factor-beta (TGF- $\beta$ ) signaling, and response to drugs. It was obvious that these pathways are closely associated with cancer development and metastasis (Fig. 5). Additionally, these results coincide with most recent results that indicate that Mkp-1 promotes angiogenesis and metastasis in lung cancer [52] and provide useful information for further research on the detailed molecular mechanisms of Mkp-1 in CRC development.

Networks identified hub nodes and interactions, which may aid in elucidating the underlying molecular mechanisms. The constructed PPI network based on STRING included 194 nodes and 132 edges. Nine DEGs were identified with degree $>5$. These genes regulated by Mkp-1 were regarded as hub genes, and may play important roles in tumor development (Table 1). The results of this study revealed that the molecular processes regulated by these candidate genes are enriched in tissue development, positive regulation of chemokine production, cell differentiation, positive regulation of chemokines, positive regulation of cytokine secretion, organ morphogenesis, regulation of signal transduction, positive regulation of cell motility, and extracellular matrix organization. Moreover, the pathways identified in KEGG were connected to legionellosis, IL-17 signaling pathway, TNF signaling pathway, and proteoglycans in cancer (Table 1). These results are in agreement with previously published articles that showed that Mkp-1 involved in the regulation of the immune response, cell proliferation, and cell differentiation through its role in MAPK signaling pathways [12, 28].
We also found that some of these genes have been used as diagnostic biomarkers for CRC and other types of tumors; in particular, overexpression of Tagln is associated with the progression of colon cancer and may serve as a new biomarker for predicting the progression and prognosis of CRC [26, 53]. A previous study has shown that Postn expression in cancer cells promotes metastasis of CRC, by activating the PI3 kinase (PI3K)/ protein kinase $B$ (Akt) signaling pathway, which supported our results that showed that Mkp-1 may influence tumor progression and metastasis $[54,55]$ through regulation of Postn expression; Postn could also be used as a prognostic and predictive factor for CRC [54]. Mkp1 induces CRC metastasis by regulating the expression of the Itga8 gene, a member of the integrin family pattern recognition receptors that participate in many cellular pathways, including adhesion and metastatic spread of tumor cells [56]. In addition, Itga8 expression may be a potential diagnostic marker of colon cancer [57]. Recent studies have shown that there is a correlation between early brain metastasis of lung cancer and the Acta2 gene mutation, and Acta 2 might be a promising diagnostic and prognostic target for lung [58] and colon cancers [59]. More recent studies have shown that Nr5a2, the last one of the candidate genes in our study, controls the development of some types of cancer by promoting cancer cell proliferation by regulating cell cycle. In particular, Nr5a2 knockdown in colon cancer cell lines leads to an impairment in cell proliferation $[60,61]$.

These results indicate that hub genes are associated with cancer development; therefore, we performed survival analyses for these genes to explore their prognostic 
value in the survival of patients with colon cancer in different cohort studies. We identified a higher expression of candidate genes leads to poor survival in CRC patients. Overall, these findings suggest that hub genes may play a critical role in cancer development and may be a useful marker for predicting the survival of cancer patients and may be significant in determining treatment outcomes.

\section{Conclusion}

Our study revealed that there are differences in molecular pathways between different stages of CRC. Therefore, our findings regarding differences in DEG between adenomas and carcinomas and their molecular targets involved in this study could help in early determination of the adenoma stage and may decrease or prevent CRC progression. Additionally, Mkp-1 plays a crucial role in CRC tumorigenesis by remodeling pathways related to cell proliferation, metastasis, and immune responses. Moreover, by considering their limitations, we suggest that hub genes identified in this work may be used in the prognosis of CRC. Functional investigations of the mechanisms related to these genes are necessary.

\section{Abbreviations \\ CRC: Colorectal cancer; Mkp-1: Mitogen-activated protein kinase phosphatase-1; RNA seq: RNA sequencing; KO: Knockout; AOM: Azoxymethane; DSS: Dextran sodium sulfate; DEGs: Differentially expressed genes; GO: Gene Ontology; KEGG: Kyoto Enrichment of Genes and Genomes; PPI: Protein protein interaction; TGF-beta: Transforming growth factor beta; ECM: Extracellular matrix; WT: Wild type; AS: Adenoma stage; CS: Carcinoma stage; H\&E: Hematoxylin and eosin; C: Control; FPKM: Kilobase of exon model per Million mapped reads; DAVID: The NIH Database for Annotation, Visualization and /??/Integrated Discovery; Tnf: Tumor necrosis factor B; Postn: Periostin; Itga8: Integrin alpha-8; Acta2: Actin, aortic smooth muscle; Tagln: Transgelin; Cxcl1: Growth-regulated alpha protein; Ogn: Mimecan; Eno3: Beta-enolase; Nr5a2: Nuclear receptor subfamily 5 group A member 2}

\section{Supplementary Information}

The online version contains supplementary material available at https://doi. org/10.1186/s12885-021-08200-0.

\section{Additional file 1. ARRIVE Guidelines checklist. \\ Additional file 2: sFig 1. Volcano plots and sFig 2. Survival curves, Prognostic values of some candidate genes in CRC different cohort.}

Additional file 3. The list of DEGs for adenoma stage in wild type and Mkp-1 knockout mice.

Additional file 4. The list of DEGs for carcinoma stage in wild type and Mkp-1 knockout mice.

Additional file 5. The enrichment analysis for common DEGs between wild type and Mkp-1 knockout mice.

Additional file 6. The list of GO terms and KEGG pathways wild type and Mkp-1 knockout mice.

Additional file 7. The survival analysis of the overall and disease-free survival of colorectal cancer patients based on the PrognoScan database.

\section{Institutional review board statement}

All animal experiments were performed with the approval of the Laboratory Animal Ethics Committee of Zhejiang University. Animal studies are conducted in compliance with the ARRIVE guidelines. All the studies were performed in accordance with relevant guidelines and regulations. A complete ARRIVE Guidelines checklist is included in Additional file 1.

\section{Authors' contributions}

Zhao-Hong Zheng conducted all experimentation. Ahmed Hammad conducted bioinformatics analysis and wrote the manuscript. Akhileshwar Namani and Mohamed Elshaer helped in data curation. Xiu Jun Wang conceived the study, provided data, experimental design, and helped in writing the manuscript. Xiuwen Tang conceived the study and directed it, and wrote the manuscript. All authors agreed to be accountable for all aspects of the work. All authors read and approved the final manuscript.

\section{Funding}

This work was supported by the National Natural Science Foundation of China (31971188 and 31370772).

\section{Availability of data and materials}

The RNA-seq data of raw and processed files have been deposited in NCBI Gene Expression Omnibus, accession number GSE164960 (https://www.ncbi. nlm.nih.gov/geo/query/acc.cgi?acc=GSE164960).

\section{Declarations}

Ethics approval and consent to participate

All animal experiments were performed with the approval of the Laboratory Animal Ethics Committee of Zhejiang University.

\section{Consent for publication}

Not applicable.

\section{Competing interests}

The authors declare that they have no competing interests.

\section{Author details}

${ }^{1}$ Department of Biochemistry and Department of Thoracic Surgery of The First Affiliated Hospital, Zhejiang University School of Medicine, Hangzhou 310003, People's Republic of China. ${ }^{2}$ Department of Pharmacology, Zhejiang University School of Medicine, Zhejiang University, Hangzhou 310058, People's Republic of China. ${ }^{3}$ Present address: Department of Biotechnology, Institute of Science, GITAM, Visakhapatnam 530045, India.

Received: 7 January 2021 Accepted: 13 April 2021

Published online: 25 May 2021

\section{References}

1. Siegel R, Naishadham D, Jemal A. Cancer statistics, 2013. CA Cancer J Clin. 2013;63(1):11-30. https://doi.org/10.3322/caac.21166.

2. Sadeghi MR, Jeddi F, Soozangar N, Somi MH, Samadi N. The role of Nrf2Keap1 axis in colorectal cancer, progression, and chemoresistance. Tumor Biol. 2017:39:1010428317705510.

3. Chen W, Zheng R, Baade PD, Zhang S, Zeng H, Bray F, et al. Cancer statistics in China, 2015. CA Cancer J Clin. 2016;66(2):115-32. https://doi.org/10.3322/ caac.21338.

4. Chirica M, Leconte M, Oberlin O, Dousset B. Cancers colorectaux : traitement chirurgical des métastases hépatiques. Presse Med. 2012;41(1): 58-67. https://doi.org/10.1016/j.lpm.2011.10.009.

5. Balch C, Ramapuram JB, Tiwari AK. The epigenomics of embryonic pathway signaling in colorectal cancer. Front Pharmacol. 2017;8. https://doi.org/10.33 89/fphar.2017.00267.

6. Riihimäki $M$, Hemminki A, Sundquist J, Hemminki K. Patterns of metastasis in colon and rectal cancer. Sci Rep. 2016;6(1):29765. https://doi.org/10.1038/ srep29765.

7. Lian W, Jin H, Cao J, Zhang X, Zhu T, Zhao S, et al. Identification of novel biomarkers affecting the metastasis of colorectal cancer through bioinformatics analysis and validation through aRT-PCR. Cancer Cell Int. 2020;20(1):105. https://doi.org/10.1186/s12935-020-01180-4. 
8. Duffy MJ. Personalized treatment for patients with colorectal cancer: role of biomarkers. Biomark Med. 2015;9(4):337-47. https://doi.org/10.221 7/bmm.15.3.

9. Zhu Y, Gu L, Li Y, Lin X, Shen H, Cui K, et al. miR-148a inhibits colitis and colitis-associated tumorigenesis in mice. Cell Death Differ. 2017;24(12):2199209. https://doi.org/10.1038/cdd.2017.151.

10. Terzić J, Grivennikov S, Karin E, Karin M. Inflammation and colon cancer. Gastroenterology. 2010;138:2101-2114.e2105.

11. Feagins LA, Souza RF, Spechler SJ. Carcinogenesis in IBD: potential targets for the prevention of colorectal cancer. Nat Rev Gastroenterol Hepatol. 2009;6:297.

12. Low HB, Zhang Y. Regulatory roles of MAPK phosphatases in cancer. Immune Netw. 2016;16(2):85-98. https://doi.org/10.4110/in.2016.16.2.85.

13. Li J, Wang H, Zheng Z, Luo L, Wang P, Liu K, et al. Mkp-1 cross-talks with $\mathrm{Nrf2/Ho-1}$ pathway protecting against intestinal inflammation. Free Radic Biol Med. 2018;124:541-9. https://doi.org/10.1016/j.freeradbiomed.2 018.07.002.

14. Liu Y, Shepherd EG, Nelin LD. MAPK phosphatases — regulating the immune response. Nat Rev Immunol. 2007;7(3):202-12. https://doi.org/10.1 038/nri2035.

15. Dubey NK, Peng B-Y, Lin C-M, Wang PD, Wang JR, Chan C-H, et al. NSC 95397 suppresses proliferation and induces apoptosis in colon cancer cells through MKP-1 and the ERK1/2 pathway. Int J Mol Sci. 2018;19(6):1625. https://doi.org/10.3390/ijms19061625.

16. Loda M, Capodieci P, Mishra R, Yao H, Corless C, Grigioni W, et al. Expression of mitogen-activated protein kinase phosphatase- 1 in the early phases of human epithelial carcinogenesis. Am J Pathol. 1996;149:1553-64.

17. Montagut C, Iglesias M, Arumi M, Bellosillo B, Gallen M, Martinez-Fernandez A, et al. Mitogen-activated protein kinase phosphatase-1 (MKP-1) impairs the response to anti-epidermal growth factor receptor (EGFR) antibody cetuximab in metastatic colorectal cancer patients. Br J Cancer. 2010;102(7): 1137-44. https://doi.org/10.1038/sj.bjc.6605612.

18. Lee M, Young Kim S, Kim J, Kim H-S, Kim S-M, Kim EJ. Mitogen-activated protein kinase phosphatase-1 inhibition and sustained extracellular signalregulated kinase $1 / 2$ activation in camptothecin-induced human colon cancer cell death. Cancer Biol Ther. 2013;14(11):1007-15. https://doi.org/1 0.4161/cbt.26044.

19. Keyse SM. Dual-specificity MAP kinase phosphatases (MKPs) and cancer. Cancer Metastasis Rev. 2008;27(2):253-61. https://doi.org/10.1007/s10555008-9123-1.

20. Smallie T, Ross EA, Ammit AJ, Cunliffe HE, Tang T, Rosner DR, et al. Dualspecificity phosphatase 1 and tristetraprolin cooperate to regulate macrophage responses to lipopolysaccharide. J Immunol (Baltimore, Md : 1950). 2015;195:277-88.

21. McGrath JC, Lilley E. Implementing guidelines on reporting research using animals (ARRIVE etc.): new requirements for publication in BJP. $\mathrm{Br} J$ Pharmacol. 2015;172(13):3189-93. https://doi.org/10.1111/bph.12955.

22. Kilkenny C, Browne W, Cuthill IC, Emerson M, Altman DG, Group NCRR GW. Animal research: reporting in vivo experiments: the ARRIVE guidelines. Br J Pharmacol. 2010;160(7):1577-9. https://doi.org/10.1111/ j.1476-5381.2010.00872.x.

23. De Robertis M, Massi E, Poeta ML, Carotti S, Morini S, Cecchetelli L, et al. The AOM/DSS murine model for the study of colon carcinogenesis: from pathways to diagnosis and therapy studies. J Carcinog. 2011;10:9-9.

24. Guo Y, Su Z-Y, Zhang C, Gaspar JM, Wang R, Hart RP, et al. Mechanisms of colitis-accelerated colon carcinogenesis and its prevention with the combination of aspirin and curcumin: Transcriptomic analysis using RNAseq. Biochem Pharmacol. 2017;135:22-34. https://doi.org/10.1016/j.bcp.2017. 02.021.

25. Suzuki R, Kohno H, Sugie S, Tanaka T. Sequential observations on the occurrence of preneoplastic and neoplastic lesions in mouse colon treated with azoxymethane and dextran sodium sulfate. Cancer Sci. 2004:95(9):7217. https://doi.org/10.1111/j.1349-7006.2004.tb03252.x.

26. Hammad A, Zheng Z-H, Gao Y, Namani A, Shi H-F, Tang X. Identification of novel Nrf2 target genes as prognostic biomarkers in colitis-associated colorectal cancer in Nrf2-deficient mice. Life Sci. 2019;238:116968. https:// doi.org/10.1016/j.lfs.2019.116968.

27. Khor TO, Huang M-T, Kwon KH, Chan JY, Reddy BS, Kong A-N. Nrf2-deficient mice have an increased susceptibility to dextran sulfate sodium-induced colitis. Cancer Res. 2006;66(24):11580-4. https://doi.org/10.1158/0008-5472. CAN-06-3562.
28. Zheng Z, Chen Y, Huang J, Deng H, Tang X, Wang XJ. Mkp-1 is required for chemopreventive activity of butylated hydroxyanisole and resveratrol against colitis-associated colon tumorigenesis. Food Chem Toxicol. 2019; 127:72-80. https://doi.org/10.1016/j.fct.2019.02.044.

29. Trapnell C, Williams BA, Pertea G, Mortazavi A, Kwan G, van Baren MJ, et al. Transcript assembly and quantification by RNA-Seq reveals unannotated transcripts and isoform switching during cell differentiation. Nat Biotechnol. 2010;28(5):511-5. https://doi.org/10.1038/nbt.1621.

30. Leng N, Dawson JA, Thomson JA, Ruotti V, Rissman Al, Smits BM, et al. EBSeq: an empirical Bayes hierarchical model for inference in RNA-seq experiments. Bioinformatics. 2013;29(8):1035-43. https://doi.org/10.1093/ bioinformatics/btt087.

31. Khan A, Mathelier A. Intervene: a tool for intersection and visualization of multiple gene or genomic region sets. BMC Bioinformatics. 2017;18(1):287. https://doi.org/10.1186/s12859-017-1708-7.

32. Huang DW, Sherman BT, Lempicki RA. Systematic and integrative analysis of large gene lists using DAVID bioinformatics resources. Nat Protoc. 2009;4:44-57.

33. Szklarczyk D, Franceschini A, Wyder S, Forslund K, Heller D, Huerta-Cepas J, et al. STRING v10: protein-protein interaction networks, integrated over the tree of life. Nucleic Acids Res. 2015;43(D1):D447-52. https://doi.org/10.1093/ nar/gku1003.

34. Shannon P, Markiel A, Ozier O, Baliga NS, Wang JT, Ramage D, et al. Cytoscape: a software environment for integrated models of biomolecular interaction networks. Genome Res. 2003;13(11):2498-504. https://doi.org/1 $0.1101 /$ gr. 1239303

35. Babicki S, Arndt D, Marcu A, Liang Y, Grant JR, Maciejewski A, et al. Heatmapper: web-enabled heat mapping for all. Nucleic Acids Res. 2016;44:17

36. Goswami CP, Nakshatri H. PROGgeneV2: enhancements on the existing database. BMC Cancer. 2014;14(1):970. https://doi.org/10.1186/1471-24 07-14-970.

37. Mizuno H, Kitada K, Nakai K, Sarai A. PrognoScan: a new database for metaanalysis of the prognostic value of genes. BMC Med Genet. 2009;2(1):18 https://doi.org/10.1186/1755-8794-2-18.

38. Kanehisa M, Goto S. KEGG: kyoto encyclopedia of genes and genomes. Nucleic Acids Res. 2000;28(1):27-30. https://doi.org/10.1093/nar/28.1.27.

39. Kanehisa M. Toward understanding the origin and evolution of cellular organisms. Protein Sci. 2019;28(11):1947-51. https://doi.org/10.1002/pro.3715.

40. Kanehisa M, Furumichi M, Sato Y, Ishiguro-Watanabe M, Tanabe M. KEGG: integrating viruses and cellular organisms. Nucleic Acids Res. 2021;49(D1): D545-51. https://doi.org/10.1093/nar/gkaa970.

41. Nelson WJ, Nusse R. Convergence of Wnt, beta-catenin, and cadherin pathways. Science. 2004;303(5663):1483-7. https://doi.org/10.1126/science.1 094291.

42. Segditsas S, Tomlinson I. Colorectal cancer and genetic alterations in the Wnt pathway. Oncogene. 2006;25(57):7531-7. https://doi.org/10.1038/sj. onc. 1210059 .

43. Grivennikov SI. Inflammation and colorectal cancer: colitis-associated neoplasia. Semin Immunopathol. 2013;35(2):229-44. https://doi.org/10.1007/ s00281-012-0352-6.

44. Caiazza F, Ryan EJ, Doherty G, Winter DC, Sheahan K. Estrogen receptors and their implications in colorectal carcinogenesis. Front Oncol. 2015;5:19.

45. Tattoli I, Sorbara MT, Philpott DJ, Girardin SE. Bacterial autophagy: the trigger, the target and the timing. Autophagy. 2012;8(12):1848-50. https:// doi.org/10.4161/auto.21863.

46. Wu Y, Yao J, Xie J, Liu Z, Zhou Y, Pan H, et al. The role of autophagy in colitis-associated colorectal cancer. Signal Transduct Target Ther. 2018;3(1): 31. https://doi.org/10.1038/s41392-018-0031-8.

47. Wood PA, Yang X, Hrushesky WJM. The role of circadian rhythm in the pathogenesis of colorectal cancer. Curr Colorectal Cancer Rep. 2010;6(2):7482. https://doi.org/10.1007/s11888-010-0045-2.

48. Ríos-Arrabal S, Muñoz-Gámez JA, Jiménez-Ruíz SM, Casado-Ruíz J, ArtachoCordón F, León-López J. Circadian regulation of colon cancer stem cells: implications for therapy, colorectal cancer - from pathogenesis to treatment. In: Rodrigo L, editor. Colorectal Cancer. Rijeka: IntechOpen; 2016. p. 23-50.

49. Karantanos T, Theodoropoulos G, Pektasides D, Gazouli M. Clock genes: their role in colorectal cancer. World J Gastroenterol. 2014;20(8):1986-92. https:// doi.org/10.3748/wjg.v20.i8.1986.

50. Battaglin F, Puccini A, Intini R, Schirripa M, Ferro A, Bergamo F, et al. The role of tumor angiogenesis as a therapeutic target in colorectal cancer. 
Expert Rev Anticancer Ther. 2018;18(3):251-66. https://doi.org/10.1080/1473 7140.2018 .1428092$.

51. Ding $\mathrm{X}$, Duan $\mathrm{H}$, Luo $\mathrm{H}$. Identification of core gene expression signature and key pathways in colorectal cancer. Front Genet. 2020;11. https://doi.org/1 0.3389/fgene.2020.00045.

52. Moncho-Amor V, Ibañez de Cáceres I, Bandres E, Martínez-Poveda B, Orgaz JL, Sánchez-Pérez I, et al. DUSP1/MKP1 promotes angiogenesis, invasion and metastasis in non-small-cell lung cancer. Oncogene. 2011;30(6):668-78. https://doi.org/10.1038/onc.2010.449.

53. Zhang $Y$, Ye $Y$, Shen $D$, Jiang $K$, Zhang $H$, Sun $W$, et al. Identification of transgelin-2 as a biomarker of colorectal cancer by laser capture microdissection and quantitative proteome analysis. Cancer Sci. 2010;101(2): 523-9. https://doi.org/10.1111/j.1349-7006.2009.01424.x.

54. Xu X, Chang W, Yuan J, Han X, Tan X, Ding Y, et al. Periostin expression in intra-tumoral stromal cells is prognostic and predictive for colorectal carcinoma via creating a cancer-supportive niche. Oncotarget. 2016;7(1): 798-813. https://doi.org/10.18632/oncotarget.5985.

55. Bao S, Ouyang G, Bai X, Huang Z, Ma C, Liu M, et al. Periostin potently promotes metastatic growth of colon cancer by augmenting cell survival via the Akt/PKB pathway. Cancer Cell. 2004;5(4):329-39. https://doi.org/10.1 016/S1535-6108(04)00081-9.

56. Schwartz MA, Ginsberg MH. Networks and crosstalk: integrin signalling spreads. Nat Cell Biol. 2002;4:E65. https://doi.org/10.1038/ncb0402-e65.

57. Gong YZ, Ruan GT, Liao XW, Wang XK, Liao C, Wang S, et al. Diagnostic and prognostic values of integrin a subfamily mRNA expression in colon adenocarcinoma. Oncol Rep. 2019;42(3):923-36. https://doi.org/10.3892/or.2 019.7216.

58. Lee HW, Park YM, Lee SJ, Cho HJ, Kim D-H, Lee J-I, et al. Alpha-Smooth muscle actin (ACTA2) is required for metastatic potential of human lung adenocarcinoma. Clin Cancer Res. 2013;19(21):5879-89. https://doi.org/10.11 58/1078-0432.CCR-13-1181.

59. Zhao B, Baloch Z, Ma Y, Wan Z, Huo Y, Li F, et al. Identification of potential key genes and pathways in early-onset colorectal cancer through bioinformatics analysis. Cancer Control. 2019;26:1073274819831260.

60. Liu L, Li Y, Pan B, Zhang T, Wei D, Zhu Y, et al. Nr5a2 promotes tumor growth and metastasis of gastric cancer AGS cells by Wnt/beta-catenin signaling. OncoTargets Ther. 2019;12:2891-902. https://doi.org/10.2147/OTT. S201228.

61. Bayrer JR, Mukkamala S, Sablin EP, Webb P, Fletterick RJ. Silencing LRH-1 in colon cancer cell lines impairs proliferation and alters gene expression programs. Proc Natl Acad Sci. 2015;112(8):2467-72. https://doi.org/10.1073/ pnas. 1500978112.

\section{Publisher's Note}

Springer Nature remains neutral with regard to jurisdictional claims in published maps and institutional affiliations.

Ready to submit your research? Choose BMC and benefit from:

- fast, convenient online submission

- thorough peer review by experienced researchers in your field

- rapid publication on acceptance

- support for research data, including large and complex data types

- gold Open Access which fosters wider collaboration and increased citations

- maximum visibility for your research: over $100 \mathrm{M}$ website views per year

At BMC, research is always in progress.

Learn more biomedcentral.com/submissions 